\title{
EIN NEUARTIGES ANTRIEBS- UND VERSTELLSYSTEM ZUR SICHEREN PLAZIERUNG VON BIOPSIENADELN IM MAMMAGEWEBE
}

\author{
G. Grimm, O. Wendt, U. Bocnick
}

Technische Universität Berlin, Institut für Mikrotechnik und Medizintechnik

\section{I:INLEITUNG}

In der Diagnostik des Mammakarzinoms werden die Mammographic und Sonographie vor allem durch die hochauflösende Magnetresonanztomographic unterstützt [2]. Ist für die weitere Diagnostik oder Therapieplanung eine Mammabiopsie indiziert, sollte sie durch das bildgebende Verfahren gestützt werden, das die Läsion am besten darstellt [1]. Durch die technische Weiterentwicklung der o.g. bildgebenden Verfahren gelingt es jetzt immer besser auch Tumoren von wenigen Millimetern im Durchmesser darzustellen [3]. Leider hat sich die Treffsicherheit der Biopsiesysteme nicht parallel dazu entwickelt, so dass eine geschlossene MRgeführte Biopsie erst ab einer Tumorgröße von $10 \mathrm{~mm}$ durchgeführt werden sollte [4].

Gefördert durch die Deutsche Krebshilfe wurde am Institut für Mikrotechnik und Medizintechnik der Technischen Universität Berlin in enger Zusammenarbeit mit Kliniken der Charité Berlin ein neuartiges Biopsiesystem für histologische Untersuchungen von Mammatumoren im Frühstadium ( $<5 \mathrm{~mm}$ ) entwickelt, das eine vollautomatische Mammabiopsie direkt im Isozentrum eines Kernspintomographen ermöglicht, Die Steuerung und Überwachung der automatisierten Biopsie erfolgt durch einen Steuercomputer, der über Lichtwellenleiter mit dem Biopsiesystem verbunden ist. Für dieses Biopsiesystem wird ein Antriebs- und Verstellsystem vorgestellt, das eine stufenlose Einstellung der Eindringtiefe der Biopsienadel in das Mammagewebe und den Vorschub des Schussapparates auf einen halben Millimeter genau ermöglicht.

\section{MATERIALIEN UND METHODEN}

Es wurde ein Antriebs- und Verstellsystem entwickelt, das in das o. g. Biopsiesystem integriert und in einem Kernspintomographen eingesetzt werden kann, Abb. 1. Dabei mussten das starke Magnetfeld eines 1,5-Tesla MR-Tomographen und die vorhandenen Anschlussbedingungen des Schussapparates sowie des Positionierungs- und Navigationssystems berücksichtigt werden. Der für die Integration in das Biopsiesystem zur Verfügung stehende Raum betrug $163 \times 46 \times 34 \mathrm{~mm}^{3}$. Die Positioniergenauigkeit der Biopsienadel von $\pm 0,5 \mathrm{~mm}$ bei einer Eindringtiefe von mindestens $55 \mathrm{~mm}$ mussten dabei ebenso berücksichtigt werden, wie eine konstante Vorschubgeschwindigkeit des Schussapparates, die ca. $0,2-1 \mathrm{~m} / \mathrm{s}$ betragen sollte. Dic Bewegungs- und Kräfteverhältnisse am Antriebs- und Verstellsystem wurden analytisch bestimmt und die Ergebnisse anhand eines vereinfachten Modellsystems evaluiert.

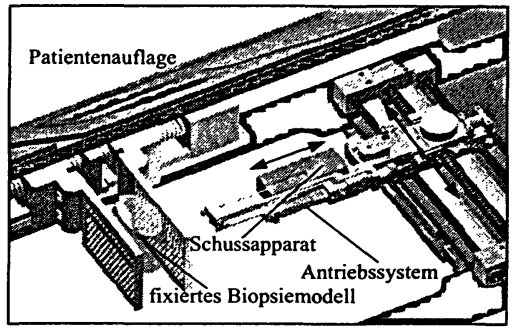

Abbildung 1: Ausschnitt des Biopsiesystems mit dem integrierten Antriebs- und Verstellsystem

\section{ERGEBNISSE}

Das Antriebs- und Verstellsystem besteht aus dem Antriebs- und Verstellzylinder und den Verbindungselementen, die eine Ankopplung des Zylinders an das Biopsiesystem ermöglichen. In dem Zylinder läuft ein pneumatisch angetriebener Verstellkolben, der über die Kolbenstange mit dem Schussapparat verbunden ist. Der Vorschubweg des Verstellkolbens wird durch einen hydraulisch angetriebenen Anschlagkolben begrenzt. Das Hydraulikfluid wird dafür über die entsprechend gestaltete Kolbenstange in den Zylinderinnenraum zwischen beide Kolben eingebracht und drückt so den Anschlagkolben an die geforderte Anschlagposition. Wird die Hydraulikzufuhr geschlossen und die Druckluftzufuhr für den Verstellkolben geöffnet, werden beide Kolben und das sich dazwischen befindende Hydraulikfluid um den eingestellten Weg verschoben. Abb. 2 zeigt den Prototypen des Antriebs- und Verstellzylinders im Schnitt, um das Funktionsprinzip zu verdeutlichen. Über einen weiteren Druckluftanschluss, der sich auf der dem Verstellkolben gegenüberliegenden Seite befindet, kann das System wahlweise entweder wieder in die Ausgangsposition gebracht oder für eine erneute Biopsie mit gleicher Eindringtiefe zurückgesetzt werden. Das vollständige Antriebs- und Verstellsystem ist in Abb. 3 dargestellt.

\section{Bereitgestellt von | Technische Universität Berlin}




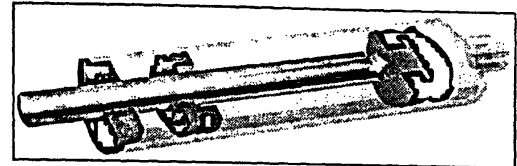

Abbildung 2: Schnitt durch den Prototypen des Antriebs- und Verstellzylinders

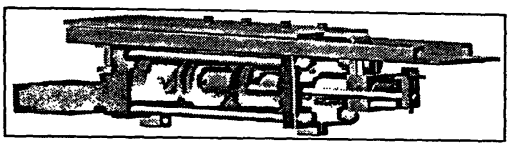

Abbildung 3: Ein neu entwickeltes Antriebs- und Verstellsystem zur sicheren Plazierung von Biopsienadeln im Mammagewebe

Der Schussapparat wird auf der Oberseite des in Abb. 3 gezeigten Systems befestigt. Die Wegmessung für die Verstellung des Anschlagkolbens erfolgt durch einen optischen Wegsensor, der in Verbindung mit einem reflektierenden Raster ein inkrementales Wegmesssystem bildet. Dieses Raster besteht aus einer reflektierenden Folie, auf die eine mit schwarzem Gitter bedruckte Klarsichtfolie geklebt ist. Die Striche des Gitters haben eine Stärke von 0,5 mm und liegen jeweils $0,5 \mathrm{~mm}$ auseinander. Damit entspricht die Gitterauflösung der geforderten Genauigkeit von $\pm 0,5 \mathrm{~mm}$. Der Wegsensor liefert der Steuerungseinheit des Biopsiesystems elektrische Impulse, wenn er über das Raster bewegt wird. Nach Anpassung der Steuerungseinheit an die gewählte Gitterauflösung von $0,5 \mathrm{~mm}$ berechnet diese anhand der gezähiten Impulse den zurückgelegten Weg und öffnet bzw. schließt die Ventile der einzelnen Druckluft- bzw. Hydraulikleitungen.

Diagramm 1 zeigt den analytisch ermittelten Druck, der am Verstellkolben anliegen muß, damit der Schussapparat mit einer konstanten Geschwindigkeit von $0,5 \mathrm{~m} / \mathrm{s}$ vorgeschoben wird, nachdem die Biopsienadel in das Mammagewebe eingedrungen ist.

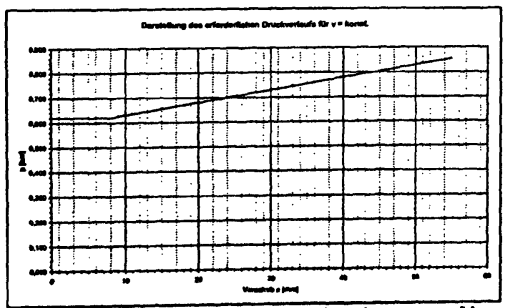

Diagramm 1: Druckverlauf für eine konstante Vorschubgeschwindigkeit des Schussapparates mit $p=f(x)$
Diagramm 2 zeigt, dass diese Geschwindigkeit nach einem Beschleunigungsweg von ca. $8 \mathrm{~mm}$ erreicht ist. Dieser Weg entspricht vor der Punktion gerade dem Abstand der Biopsienadel zum Mammagewebe.

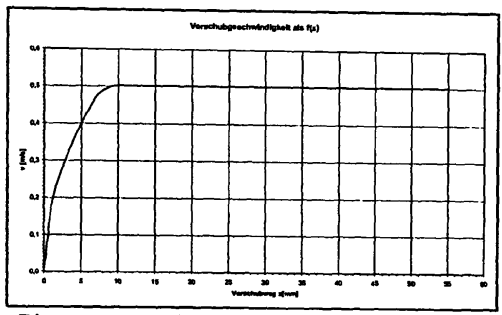

Diagramm 2: Verlauf der Vorschubgeschwindigkeit des Schussapparates in Abhängigkeit vom Vorschubweg $\mathrm{X}$

Die Kurven wurden nach folgenden Formeln erstellt:

1. für $0<x \leq 8 \mathrm{~mm}$ :

$$
\begin{aligned}
& v_{\text {SchuB }}(x)=\sqrt{\frac{2 \cdot\left(p_{1} \cdot A_{K}-F_{R}\right)}{m_{\text {Schuss }}} \cdot x} \\
& p_{1}(x)=\frac{1}{A_{K}}\left(\frac{v^{2} \cdot m_{\text {Schuss }}}{2 x}+F_{R}\right), \text { mit } v=0,5 \frac{\mathrm{m}}{\mathrm{s}}
\end{aligned}
$$

2. für $8<x \leq 55 \mathrm{~mm}$ muß der anzulegende Druck $p_{1}$ linear zum Gewebewiderstand $F_{G}$ um den Wert $p_{2}=F_{G} / A_{K}$ ansteigen, damit die Geschwindigkeit konstant bleibt. $F_{G}$ wurde experimentell ermittelt zu:

$F_{G}=\frac{2,5}{16} \cdot x_{G}$, mit $F_{G}$ in $[N]$ und $x_{G}$ in $[\mathrm{mm}]$.

$\mathbf{x}_{\mathrm{G}}=$ Gewebeeindringtiefe; $A_{K}=$ Kolbenfläche;

$\mathrm{m}_{\text {Schuss }}=$ Masse d. Schussapparates; $F_{R}=$ Reibungskräfte; $p=p_{1}+p_{2}=$ relat. Druck am Verstellkolben

\section{LITERATURHINWEISE}

[1] Heywang-Köbrunner, Sylvia H., Schreer, Ingrid: Bildgebende Mammadiagnostik. Georg Thieme Verlag, 1996

[2] Keßler M, Milz P, Sittek H, Reiser M. Bildyebende Verfahren zur Diagnostik und Differentialdiagnostik des Mammakurzinoms. Bildgebung 1995; 62; 160 - 172

[3] Oellinger H, Blohmer J U, Stahl II. Das Mammakarzinom: Diagnostik und Therapic. Skript der Charitê Campus Virchow Klinikum Strahlenklinik und Poliklinih

[4] Fischer U, Rodenwaldt J, Ilundertmatk C. DSler W: Grabbe E.

AIRT-gestiutzte Biopsic und Lokulinution der Akmma. Radiologe 1997: 37: 692-701 